\title{
Proof-of-Concept for 2D/CT Element Analysis of Entire Cryofrozen Islets of Langerhans using a Cryoloop Synchrotron X-ray Fluorescence Setup
}

\author{
Authors \\ 1,2*Björn De Samber, ${ }^{3}$ Mohammed Bensellam, ${ }^{1,4}$ Stijn J.M. Van Malderen, \\ ${ }^{4}$ Frank Seiboth, ${ }^{4,5}$ Dennis Brückner, ${ }^{4}$ Jan Garrevoet, ${ }^{4}$ Gerald Falkenberg, \\ ${ }^{3}$ Jean-Christophe Jonas, ${ }^{1}$ Laszlo Vincze \\ ${ }^{1}$ X-ray Microspectroscopy and Imaging group (XMI), Department of Analytical Chemistry, Ghent \\ University, Ghent, Belgium \\ 2 imec - Vision Lab, University of Antwerp, Wilrijk, Belgium \\ ${ }^{3}$ Université Catholique de Louvain (UCL), Institut de recherche expérimentale et clinique, Pôle \\ d'endocrinologie, diabète et nutrition, Brussels, Belgium \\ ${ }^{4}$ Deutsches Elektronensynchrotron (DESY), Hamburg, Germany \\ ${ }^{5}$ University of Hamburg, Department of Physics, Hamburg, Germany \\ *Both authors contributed equally to this study
}

Contact: bjorn.desamber@uantwerpen.be

\section{Keywords}

Synchrotron, XRF, islets of Langerhans, beta cells, diabetes, cryostream, tomography 


\author{
Abbreviations \\ 2D: two-dimensional \\ CRLs: compound refractive lenses \\ MLEM: maximum likelihood expectation maximization algorithm \\ CT: computed tomography \\ KO: Mt1-Mt2 double knockout \\ SR-XRF: synchrotron radiation based X-ray fluorescence \\ SDD: silicon drift detector \\ WT: wild type \\ LOD: limit of detection \\ RGB: Red Green Blue
}




\section{Abstract}

This work reports on synchrotron X-ray fluorescence (SR-XRF) imaging of vitrified islets of Langerhans in two-dimensional and computed tomography (CT) mode, and serves as a proof-ofprinciple for preparation and SR-XRF imaging of cryoloops in a cryogenic environment. Selection of suited cryoloops and cryoprotectant solution enabled vitrification of the islets. The SR-XRF experimental setup was adapted with a cryogenic gas stream enabling analysis of cryofrozen islets and a dual silicon drift detector (SDD) configuration providing increased solid angle for XRF collection. Element distributions of $\mathrm{K}, \mathrm{Ca}, \mathrm{Fe}$ and $\mathrm{Zn}$ within single, cryofrozen islets could be obtained with $500 \mathrm{~nm}$ spatial resolution using X-ray compound refractive lenses (CRLs) with adaptive phase plate. Titanium, present as trace element in the cryoloops was used as marker signal to efficiently define the XRF scan area. Preliminary 2D cryo-analysis of wild type and Mt1Mt2-knock-out islets under low/high glucose conditions did not reveal changes in distribution and concentration of $\mathrm{Ca}$, Fe and Zn. Cryotomography XRF scans indicated aspherical islets within the cryoloop, likely due to capillary effects in the cryoloop before plunge freezing. The presence of diffracting ice crystals hampered CT reconstruction, indicating the need for further development of a routine workflow ensuring perfect vitrification. Collectively, this novel multidisciplinary approach proved to be valid and can be used for future studies to assess elemental composition of islets of Langerhans or other cell clusters/tissues under different pathophysiological conditions.

\section{Significance to JAAS}

Pancreatic beta-cells within the islets of Langerhans play a key role in the pathogenesis of diabetes. Metal dyshomeostasis is involved in beta-cell pathophysiology but analytical methods for non-destructive metal imaging/quantification in whole islets in their near-native state are lacking. We performed first $\mathrm{X}$-ray nanochemical imaging on single, cryofrozen islets to investigate their elemental composition and distribution. We used cryoloops for islet capture and flash freezing, and 2D/CT XRF analysis under a cryogenic gas stream in combination with a dual SDD configuration. Our innovative method contributes significantly to the analytical techniques available to investigate the role of metals in beta-cell pathophysiology. 


\section{Introduction}

The International Diabetes Federation estimates indicate that there are 425 million people affected by diabetes around the world and this number will likely reach 629 million by 2045 . Type 2 diabetes (T2D) is the most common form of diabetes (approximately $90-95 \%$ of all diabetes cases). It arises from a complex interplay of genetic and environmental factors that affect peripheral insulin sensitivity and glucose-stimulated insulin secretion (GSIS) by pancreatic beta cells. Defective insulin secretion plays a key role in the development of the disease since the progression to frank diabetes in insulin resistant subjects only occurs when their beta cells fail to adequately compensate to meet the organism's demands ${ }^{1}$. The ensuing chronic hyperglycemia exerts further toxic effects on the beta cell differentiated phenotype, thereby leading to the worsening of the disease over time ${ }^{2,3}$. A major challenge in diabetes research is to identify and characterize the mechanisms involved in beta cell pathophysiology towards the development of strategies to preserve/restore insulin secretion in (pre)diabetic subjects.

Beta cells are located within the islets of Langerhans; compact spheroid clusters of endocrine cells with a diameter of approximately 40 to more than $500 \mu \mathrm{m}$ dispersed throughout the exocrine tissue and representing about $1 \%$ of the total pancreatic mass. In rodents, islets are mainly composed of beta cells (60-80\%) located in the core, in addition to non-beta cells located at the periphery (alpha cells, delta cells, PP or gamma cells and epsilon cells). In diabetes research, the study of beta cells within whole islets of Langerhans is required for proper evaluation of their function. Indeed, cell-cell contacts are important and dispersed beta cells display different functional responses in comparison to whole islets ${ }^{4-7}$.

Metals play fundamental roles in beta cell biology. Calcium (Ca) and potassium (K) for example modulate insulin secretion ${ }^{8-10}$, whereas zinc $(\mathrm{Zn})$ plays a vital role in insulin crystallization and storage within the secretory granules 11-13. Importantly, evidence has shown that metal dyshomeostasis, including perturbations of $\mathrm{Ca}, \mathrm{Zn}$, copper $(\mathrm{Cu})$ and iron $(\mathrm{Fe})$ are implicated in beta cell dysfunction and death, both in vitro and in animal models 10, 14-17, and correlated with T2D and its complications in humans, though further insight into this mechanism is required ${ }^{18-22 .}$ The development of novel analytical methods for non-destructive metal imaging and quantification in whole islets in their near-native state may provide novel insights into the role of metal elements in beta cell pathophysiology.

During the past years, third and even fourth generation synchrotron sources are coming online, providing new powerful capabilities for three-dimensional (3D), trace level metal imaging at micro- 
and nanometer scale ${ }^{23}$. Using the technique of synchrotron radiation induced X-ray fluorescence (SR-XRF), samples can be investigated in-depth in an essentially non-destructive manner. An accommodating trend of SR nano-XRF imaging, is the development and availability of cryogenic environments, enabling the analysis of biological samples in a vitrified state. Developments run along two lines: vacuum sample environments using a 'cold-finger' via the contact cooling principle ${ }^{24,25}$ and analysis under atmospheric conditions using a cryogenic gas stream ${ }^{26}$. The use of cryostreams has become routine in the field of protein crystallography ${ }^{27}$. In this field, the use of cryoloops has also been developed for manipulating and measuring isolated protein crystals or even crystals produced within living cells ${ }^{28}$.

In the present work, as a proof of concept study, we performed the first SR-XRF measurements on single, cryofrozen whole islets of Langerhans. Experiments took place at the P06 hard X-ray microprobe at the third-generation light source PETRAIII (Hamburg, Germany) ${ }^{29-31}$. A submicrometer $(500 \mathrm{~nm})$ sized X-ray beam was produced using compound refractive beryllium lenses with adaptive phase optics ${ }^{32}$. We devised a new, uncompromising sample preparation strategy to study islets of Langerhans by first capturing islets in dedicated cryoloops, followed by flashfreezing and two-dimensional (2D)/computed tomography (CT) XRF analysis in a cryogenic gas stream at $-150^{\circ} \mathrm{C}$. Finally, we investigated the distribution and concentration levels of zinc and other metals in wild-type (WT) and Mt1-Mt2 double knockout (KO) islets ${ }^{33}$ incubated in the presence of low (3 mM; G3) or high glucose (30 mM; G30) conditions.

\section{Materials \& Methods}

\section{$\underline{\text { Islet isolation }}$}

Pancreatic islets were isolated from male WT mice (129S1/SvImJ) and their sex and age matched KO mice (129S7/SvEvBrd-Mt1tm1Bri Mt2tm1Bri/J) by collagenase (Roche, Basel, Switzerland) digestion of the pancreas followed by density gradient centrifugation using Histopaque 1077 (Sigma, Saint-Louis, Missouri, USA). Islets were then selected with a micropipette under a stereomicroscope and precultured for 1 week in standard RPMI medium (Invitrogen, Carlsbad, CA, USA) complemented with $5 \mathrm{~g} / \mathrm{BSA}$ fraction $\mathrm{V}$ (Roche), $2 \mathrm{mM}$ glutamine (Sigma), $100 \mathrm{units} / \mathrm{ml}$ penicillin (Thermo Fisher Scientific, Waltham, Massachusetts, USA) and $100 \mu \mathrm{g} / \mathrm{ml}$ streptomycin (Thermo Fisher Scientific). Animals originated from the Jackson Laboratory (Bar Harbor, MA) and were bred in the local animal facility of the health sciences sector at UCLouvain. All experiments 
were approved by the Institutional Committee on Animal Experimentation of the Health Sciences Sector at UCLouvain under the ethics reference: Project 2017/UCL/MD/014.

\section{Sample preparation}

Sample preparation steps and images of cryo-equipment are illustrated in Fig 1a-c. Mouse islets were preincubated for $1 \mathrm{~h}$ in bicarbonate-buffered Krebs solution (KRB) $(120 \mathrm{mmol} / \mathrm{l} \mathrm{NaCl}, 4.8$ $\mathrm{mmol} / / \mathrm{KCl}, 2.5 \mathrm{mmol} / / \mathrm{CaCl}_{2}, 1.2 \mathrm{mmol} / \mathrm{l} \mathrm{MgCl}, 24 \mathrm{mmol} / \mathrm{l} \mathrm{NaHCO}, 1 \mathrm{~g} / \mathrm{l} \mathrm{BSA}$ ) containing $3 \mathrm{mM}$ glucose (G3), and then incubated for $1 \mathrm{~h}$ in KRB buffer containing G3 or G30. At the end of incubation, islets were transferred with a micropipette onto a glass slide using a binocular. Prior to this, superglue was used to fixate a hollow steel pin in the base opening of a cryocap (MD7400) and to attach the cryoloop (various specs: 200/300 $\mu \mathrm{m}$ loop diameter, 20/40 $\mu \mathrm{m}$ loop thickness, purchased from Molecular dimensions Ltd., UK) to the steel pin. The cryoloop was moved through the islet solution to capture and isolate individual islets, using a magnetic wand (MD7-411) holding the cap. After capture, islets were washed for a few seconds in a droplet of ammonium acetate buffer (150 mM, pH 7.6), (Sigma Aldrich, $\geq 99.99 \%$ trace metals basis) containing $30 \%$ glycerol (Merck, for molecular biology, $\geq 99.9 \%$ ), or pure hexadecane (Merck, for synthesis) as cryoprotectant. Immediately afterwards, cryoloops were plunge frozen in isopentane (Merck, 2-methylbutane) cooled with liquid nitrogen to $-150{ }^{\circ} \mathrm{C}$. Excess isopentane liquid on the cryoloop was blotted in the cold liquid nitrogen vapor, after which the loop was further cooled down by direct insertion in liquid nitrogen. In succession, cryoloops were covered with magnetic cryovials (MD7-402) using cryotongs for handling SPINE cryocaps (MD7-12). Using basket tongs (MD7-515), cryovials were then loaded into sample pucks (EMBL baskets, MD7-510), which were precooled in a polystyrene box filled with liquid nitrogen. Then, baskets completely filled with cryovials were transferred into the canister (MD7-511) of a dryshipper (CX100, MD7-21). Finally, dryshipper holding EMBL baskets was transported by car to P06 beamline in a dedicated shipping case (MD7-22). After analysis, all samples were stored again in the dryshipper in a reverse working manner.

\section{Experimental setup}

Synchrotron XRF experiments were performed at the P06 hard X-ray microprobe at the third generation PETRAIll synchrotron light source ${ }^{31,34}$. Beryllium compound refractive lenses (CRL) 
with correcting phase plate were deployed as focusing optics ${ }^{32}$. Additionally, prefocusing CRLs were used to prefocus the X-ray beam into the CRL lens box aperture to obtain a higher X-ray flux. A pinhole downstream of the lens box was used to clean the beam pattern. For optimal detection of elements of interest (up to bromine), a fixed excitation energy of $14 \mathrm{keV}$ was selected using a Si111 monochromator ${ }^{35}$. The sample stack was composed of (bottom-to-top order): a hexapod stage (Newport ${ }^{\mathrm{TM}}$ HXP200S-MECA) for initial alignment of the sample to the beam position, rotation stage (PiMicos ${ }^{\mathrm{TM}}$ UPR-270 Air) for XRF tomography, XY stage (Huber ${ }^{\mathrm{TM}}$ 510218.150) for centering the sample/cryoloop in the center of rotation and piezostage (Aerotech ${ }^{\mathrm{TM}}$ QNP-XY and QNP-Z combination driven by Aerotech Ensemble QLe controllers) for horizontal and vertical scanning. Scanning stage was fitted with a magnetic base mount (Hampton research, HR4-943), capable of carrying the cryocap with cryoloops. An aluminum foil covering the piezo stage and a dry nitrogen gas stream prevented the piezo scanner from icing. Two silicon drift detectors (SDDs, Vortex EM, Hitachi) fitted with aluminum collimators ( $3 \mathrm{~mm}$ aperture) were used to collect XRF photons, one at 90 degrees angle, minimizing Compton scatter, and another at a scattering angle of 75 degrees. For sample cooling, a cryostream (700 Series Cryostream Cooler, Oxford Cryosystems, UK) was positioned just above the sample, minimizing vibrations and icing.

\section{$\underline{\text { Cryogenic sample transfer }}$}

To transfer the samples onto the scanning stages of the P06 beamline, while keeping the islets vitrified, an EMBL sample basket was first transferred to a polystyrene box filled with liquid nitrogen using basket tongs, after which the box was carried into to experimental hutch and positioned close to the sample stage. The cryostream located above the sample was raised in height by approximately $5 \mathrm{~mm}$ with respect to the measuring position to facilitate quick mounting of the magnetic cryocap holding the cryoloop. A magnetic cryovial containing a sample of interest was then taken from the sample puck using a magnetic cryo-wand. Cryocap with cryoloop was then removed from the magnetic vial using long cryotongs. Cryocap with cryoloop and islet was then grasped with a different kind of cryotongs, specifically designed for transferring cryoloops from liquid nitrogen into the cryostream. Cryocap with cryoloop and islet was put on the sample stage in a fast and fluent transfer step, requiring no longer than 1-2 seconds. Then, cryotongs were opened and gently removed from the cryocap in order not to touch the cryoloop and/or sample. Immediately afterwards, cryostream was lowered $5 \mathrm{~mm}$ again to bring the cryoloop into the optimal cooling position of the cryostream, set at $120 \mathrm{~K}\left(-150^{\circ} \mathrm{C}\right)$. 


\begin{abstract}
Data analysis
Spectra were acquired by an Xspress3 pulse processor (Quantum Detectors Ltd) connected to the two Vortex EM SDDs. Energy calibration was performed based on the primary excitation energy and argon (Ar) lines. The XRF spectra of both channels underwent fitting using in-house software incorporating the fitting core of PyMCA ${ }^{36}$. All data was normalized to the ion chamber current located upstream. The sampling location of each pixel was determined as the center position of the interpolated trajectory between the encoder position when the exposure was started and ended. Each element map was created by multivariate interpolation of the point cloud of center positions, and their associated integrated peak area values for each fluorescence line. Tomographic reconstruction of the acquired sinograms was obtained via a maximum likelihood expectation maximization algorithm (MLEM) ${ }^{37}$.
\end{abstract}

\title{
Results and Discussion
}

\section{Sample preparation workflow for XRF analysis under cryogenic conditions}

The sample preparation workflow we developed to enable SR-XRF analysis of cryogenically frozen islets of Langerhans is illustrated in Fig 1a. The entire workflow involves: 1) islet culture and isolation, 2) islet capture in a cryoloop, followed by washing and application of cryoprotectant, 3 ) islet plunge freezing and blotting, 4) cryogenic transport of the samples in a dryshipper and 5) cryogenic transfer to the SR-XRF setup. Fig $1 \mathrm{~b}$ depicts a cryoloop (without mesh) glued in a hollow steel pin. The latter is glued into a cryocap holder (see Fig 1c). Although many procedures and materials for cryoloop handling are available from the protein crystallography community, a number of important optimizations were required for enabling analysis of cryofrozen islets: a) selection of proper cryoloops capable of capturing islets, b) selection of optimal cryoprotectant solution and c) adaptation of the SR-XRF setup for cryogenic analysis.

Regarding the type of cryoloops suited to capture islets (point a), we found that thicker cryoloops (e.g., $40 \mu \mathrm{m}$ loop thickness instead of $20 \mu \mathrm{m}$ loop thickness) were better suited to capture islets as they contain a larger volume of liquid. Moreover, captured islets were lost less easily from cryoloops having $200 \mu \mathrm{m}$ instead of $300 \mu \mathrm{m}$ loop diameter due to a better size match of islet and loop. Apart from empty loops, loops containing a mesh were tested, but were found less suitable as their larger contact surface exerted a greater force upon the islets. Additionally, the cryoloop mesh complicates XRF analysis of islets due to X-ray absorption effects of the mesh material. For 
a more detailed description on the type of cryoloops used, we refer to the materials and methods section. For selection of a suited cryoprotectant (point b), standard methods in cryocrystallography were tested: i) non-penetrating cryoprotectants such as mineral oil or large molecular weight polyethylene glycol (MW PEGs), ii) penetrating cryoprotectants, encompassing sugars (e.g., dextran, sucrose, glucose) and iii) alcohol-based cryoprotectants (e.g., glycerol, small MW PEGs, ethylene glycol). Oils were used undiluted while the other cryoprotectants are used at concentrations of $30 \mathrm{vol} \%$ by adjustment of the mother liquor after crystallization. In contrast with protein crystallography in which very high concentrations of cryoprotectants are required to obtain vitrified protein crystals, lower concentrations can theoretically be used for cryofixation of islets as they are kept in a medium with much lower salt concentrations, facilitating proper vitrification. In this study, we opted not to use penetrating cryoprotectants (e.g., PEG, sugars) in order not to influence the sugar-sensitive islets. In addition, polyethylene glycol-400 (PEG-400) was excluded due to osmolar pressure difference and change in islet shape. Therefore, hexadecene $\left(\mathrm{C}_{16} \mathrm{H}_{32}\right)$ - an oil often used as cryoprotectant for high pressure freezing (HPF) - and ammonium acetate buffer $\left(\mathrm{NH}_{4} \mathrm{CH}_{3} \mathrm{CO}_{2}\right)$ diluted with 30 vol\% glycerol were tested. Microscope examination of islet shape revealed that it was best preserved with hexadecene compared to $30 \%$ glycerol cryoprotectant, again likely due to stronger osmolar effects of the latter. More information on the adaption of the experimental sample stage (point $c$ ) is provided in the next section 'Experimental setup'

\section{Experimental setup for 2D/CT XRF under cryogenic conditions}

In order to subject the cryofrozen islets to XRF analysis, a custom experimental set-up compatible with 2D/CT XRF analysis was devised, shown in Fig 2a (overview) and Fig 2b (detail). Compound refractive lenses (CRLs) were used for X-ray focusing, providing an X-ray beam down to $200 \mathrm{~nm}$ with a flux of $10^{10}$ photons/s ${ }^{32}$. CRLs offer a large working distance, providing ample space around the sample for deploying a cryogenic gas stream and XRF detectors. During some scans, additional pre-focusing CRLs were inserted into the beam path, leading to a more divergent beam, higher coherence and therefore smaller spot size, albeit at the cost of X-ray flux.

The sample piezo scanner and rotation stage (framed in Fig 2a) required for 2D/CT XRF analysis were both positioned on a servo motor hexapod stage which was used to pre-align the cryoloop with islet to the beam position. Onto the piezo stage, a custom-made aluminum holder was mounted (see Fig 2a), holding a magnetic stub on which the sample cryocaps can be attached (detailed in Fig 2b). The cryostream nozzle was installed perpendicular to the sample, thereby minimizing longitudinal vibrations of the sample and dissipating the laminar flow of the cold gas 
stream equally in all horizontal directions around the sample. Temperature of the cryogenic gas flow was set to $120 \mathrm{~K}\left(-150^{\circ} \mathrm{C}\right)$ to maintain vitreous ice. In order to minimize contact of the cold gas stream with the sample stage, a centrally pierced aluminum foil was installed that diverts the cold air flow from the sample holder and piezo stack below (Fig 2b). Thermal conduction between sample and sample stage was countered using a warm, dry nitrogen flow directed onto the piezo stage, minimizing condensation effects.

A dual SDD configuration was used to increase solid angle by a factor of two compared to a single silicon drift detector (SDD) setup, enabling faster XRF scans. An additional advantage of the dual SDD configuration is its ability to observe self-absorption effects, occurring when fluorescent radiation of lower atomic number elements $(\mathrm{P}, \mathrm{S}, \mathrm{Cl}, \mathrm{K}, \mathrm{Ca})$ needs to travel across approx. 100 microns of ice in case the X-ray fluorescence is coming from the backward side of the islet ${ }^{38}$. Distance between sample and detector was kept at approx. $15 \mathrm{~mm}$ to: 1) prevent cooling of collimators/SDD detectors and 2) to reduce excessive X-ray scatter, leading to increased background in the XRF spectrum and therefore also deterioration of trace metal LODs.

In order to minimize XRF photons from the islets being absorbed by the cryoloop during 2D scanning, the cryoloop was oriented with its flat side perpendicular to the beam direction. The inset in the lower-left corner of Fig $2 b$ (Fig 2c) shows a light microscopy image of the cryofrozen islet within the cryoloop as observed by the beamline microscope. The element distributions of $\mathrm{K}$, $\mathrm{Ca}, \mathrm{Fe}$ and $\mathrm{Zn}$ of a single islet obtained by both SDDs are shown in Fig 3. Self-absorption effects can clearly be observed for the elements $\mathrm{K}$ and $\mathrm{Ca}$ : for SDD no. 0 ( $15^{\circ}$ offset from orthogonal position), element intensities are stronger on the right side of the map, whereas for SDD no. 1 (orthogonal to beam) element intensities are stronger on the left side of the map (indicated by a black dashed line). Noteworthy, bright spots are also present on the element maps of $\mathrm{K}$, Ca and Fe (indicated with a white arrow), which we believe not to be of biological origin, but likely represent contamination from the medium and/or locations where diffraction occurs. In the latter case, polycrystalline ice (present inside or on the surface of the islet) diffract X-ray photons which, on their turn, excite the surrounding environment (for example the supporting hollow steel pin of the cryoloop), causing secondary fluorescence. On the other hand, on the zinc element map, selfabsorption effects are negligible and the overall shape and morphology of the islet is clearly visible. Lower zinc intensity is present at the rim of the islet, which can be explained by the islet being thinner at this position and/or by the presence of a different kind of cells at the periphery of the islets. Indeed, beta cells, exhibiting a high zinc content, are located in the core of rodent islets, while non-beta cell are located at the periphery ${ }^{15,39}$. Typical sensitivities of the experimental set- 
up were estimated to be in the (sub) ppm range for typical scanning dwell times of $100 \mathrm{~ms}$ per point using a self-supporting pellet of NIST SRM 1577C 'bovine liver' powder.

\section{D XRF cryomapping of a whole islet of Langerhans from WT mouse.}

After optimization of the SR-XRF setup, we assessed the XRF element maps of $\mathrm{P}, \mathrm{S}, \mathrm{Cl}, \mathrm{K}, \mathrm{Ca}$, $\mathrm{Ti}, \mathrm{Fe}, \mathrm{Cu}$ and $\mathrm{Zn}$ in a WT islet incubated for $1 \mathrm{~h}$ in the presence of basal glucose condition (G3), as depicted in Fig 4. As a compromise between high resolution and short scanning time, the islet was scanned with $1 \mu \mathrm{m}$ step size and $100 \mathrm{~ms}$ dwell time. Interestingly, titanium fluorescence clearly indicates the location of the cryoloop; cryoloops are composed of a polyimide based resin 'SPIKA' with confidential composition showing ultra-low X-ray background scatter, but are indeed known to contain traces of titanium. The titanium XRF signal proved useful for fast determination of the proper sample boundaries preceding the XRF scan. For clarity, the boundary of the loop as determined from the titanium map is indicated in some element maps with a white, dashed line. Phosphorus, sulphur and chlorine element distributions contain high noise levels due to absorption in the ice layer covering the sample and sample detector distance. Chlorine showed higher signal-to-noise ratio and is present in higher concentration in the medium, between the islet and the cryoloop. Copper was close to the LOD, while the elements potassium, iron and zinc (and to a lesser extent calcium) were well above their LOD and distributed heterogeneously throughout the islets. This may stem from the heterogenous cell-type composition of the islet, as well as from the heterogenous metabolic and functional nature of beta cells ${ }^{6}$. Note that since $2 \mathrm{D}$ XRF scanning integrates the element signal across the entire depth of the sample, information on the islet morphology is masked. Therefore, in a next phase, XRF CT imaging was performed at the central height of the same islet, indicated by a white dashed line in the zinc element map in Fig 4.

\section{CT XRF on a whole islet of Langerhans from WT mouse}

For islet XRF tomography, step sizes of $500 \mathrm{~nm}$ were selected and the scanning angle range was reduced to 180 degrees to reduce scanning time (a dual SDD setup looks at the sample from both directions and enables this scanning mode). A dwell time of $50 \mathrm{~ms}$ was selected to achieve a total measuring time of approximately $12 \mathrm{~h}$. Tomographic reconstruction was performed using a maximum likelihood estimation method (MLEM) algorithm (see materials and methods section for more information). The results of the XRF CT of the WT islet are shown in Fig 5; the islet height where the virtual cross-section is obtained is indicated by a white dashed line in Fig 4. We found that the analyzed islet has an oblong shape rather than spheroid; thickest and narrowest cross- 
section through the islet was determined to be 170 and $135 \mu \mathrm{m}$, indicating a compression/elongation of about $20 \%$ of the mean value. The aspherical shape of the islet is likely caused by capillary forces exerted upon the islet in the cryoloop. Interestingly, on the left hand side panel of Fig 5, the heterogeneous distribution of zinc within the islet can clearly be discerned, together with the presence of zinc-poor areas. In the middle panel, element distributions of $\mathrm{Fe}, \mathrm{K}$, $\mathrm{Ti}, \mathrm{P}, \mathrm{Mn}$ and $\mathrm{Cu}$ are shown. Distribution of iron is similar to that of copper, with the presence of an iron hot spot, which might represent the start of iron related cell death, also referred to as ferroptosis. The phosphorus $\left(\mathrm{P}-\mathrm{K}_{\alpha}=2.015 \mathrm{keV}\right)$ and potassium $\left(\mathrm{K}-\mathrm{K}_{\alpha}=3.313 \mathrm{keV}\right)$ element distribution show the presence of a gradient across the islet, which is caused by self-absorption effects, thereby indicating that the detector signal used for this image reconstruction was oriented towards the lower part of the islet. For manganese $\left(\mathrm{Mn}-\mathrm{K}_{\alpha}=5.898 \mathrm{keV}\right)$ and $\operatorname{copper}\left(\mathrm{Cu}-\mathrm{K}_{\alpha}=\right.$ $8.047 \mathrm{keV})$, this effect was negligible, but close to the limit of detection. Titanium $\left(\mathrm{Ti}^{-} \mathrm{K}_{\alpha}=4.510\right.$ $\mathrm{keV}$ ) clearly indicates the triangular cross-section of the cryoloops. By means of an RGB representation (see Fig 5, right panel), the location of the islet relative to the cryoloop can be determined. An additional XRF cryotomography which was performed upon a KO islet under basal glucose conditions revealed an oblong islet shape as well. Due to the presence of more crystalline ice that causes strong scattering, tomographic reconstruction was strongly hampered in this case. Improvements along this line could be obtained by testing other cryoprotectant solutions and more routine sample preparation. For the reasons mentioned above (e.g. time limitation, scatter interfering with CT reconstruction), in what follows, analysis of islets under different conditions was pursued in 2D cryo-XRF mode only.

Comparative 2D XRF study between WT and KO islets exposed to basal or high glucose levels. Following XRF cryotomography, we made a preliminary assessment of element composition of islets from one WT and one $\mathrm{KO}$ mice exposed to low (3 $\mathrm{mM})$ and high (30 mM) glucose levels. Fig 6 shows zinc distribution of islets from different origin and glucose exposure: WT (left column) and $\mathrm{KO}$ mice (right column), and exposure to low (3 mM, upper row) and high glucose levels (30 $\mathrm{mM}$, lower row). All scans were performed in an identical manner, using a step size of $1 \mu \mathrm{m}$ and a dwell time of $100 \mathrm{~ms}$. For orientation purposes, the titanium element map, indicating the location of the cryoloop, is shown next to the corresponding zinc distribution. Interestingly, only the WT G3 islet shows higher maximal zinc intensities (7 kcps) compared to the other islets conditions (4 kcps), which also show a less homogeneous zinc distribution. In Fig 7, corresponding element distributions of calcium and iron are provided. For both element distributions, hot-spots are 
present, which are likely caused by the presence of microcrystals causing diffraction and secondary fluorescence (see section: 'Experimental setup for 2D/CT XRF under cryogenic conditions' for more information). Besides the fact that the calcium distribution in the islets is subject to absorption (left side of the calcium distribution in the islet is less intense than the right side), a lower calcium intensity is present in the KO-G3 islet (350 cps) compared to the other islets (600 cps). For iron, when excluding hot-spots, all islet conditions show similar maximum intensities (1400 cps), while the iron distribution is nearly homogeneous for the WT-G3 islet. Note however that 2D-XRF maps might mask the presence of heterogeneity and/or subtle change in element intensity due to variations in thickness. In order to address this problem, the mean intensity for each measured islet is provided in Fig 8a. As these intensities (expressed in cts) are normalized to the number of pixels and the Compton scatter peak (proportional to the electron density of the islet), they are proportional to the mean element concentration of each islet. Noteworthy, due to the improved signal-to-noise ratio of these islet XRF cluster spectra, elements such as $\mathrm{Mn}, \mathrm{Ni}, \mathrm{Cu} \mathrm{Se}$ and $\mathrm{Br}$ can also be studied. For completeness, a similar graph was made in which the Compton scatter normalization is discarded, thereby neglecting density differences between the different islets (see Fig 8b). 


\section{Summary \& Conclusions}

Islets of Langerhans play a key role in glucose homeostasis of the organism owing to the production of regulatory hormones, in particular the production of insulin by beta cells. Metals, on the other hand, are essential in beta cell physiology. In this context, metal dyshomeostasis has been associated with beta cell demise and the development of diabetes. Unfortunately, imaging of the trace element distribution within entire islets using fluorescent markers is hampered by insufficient sensitivity and lack of measuring the total metal pool. Synchrotron radiation based Xray fluorescence is one of the few available techniques able to provide the trace level metal distributions at the micro- and nanoscopic level at the 2D and 3D level. In order to keep the sample and its metal distributions close to the native state during XRF analysis, cryofixation of the sample is needed, after which the sample can be analysed under atmospheric conditions in a cryogenic gas stream or in-vacuum in contact with a cold-finger. The use of a cryostream can be beneficial with respect to the cold-finger approach due to its lower cost, availability of more space around the sample for detectors, optics and faster sample exchange.

In this work, we deployed CRLs with an adaptive phase plate in combination with a cryostream for 2D/CT analysis of whole, free standing and cryofrozen islets of Langerhans. Cryoloops - widely used in the field of cryocrystallography - were used for capturing, washing and plunge freezing of islets. Selection of the cryoloop and cryoprotectant (e.g., hexadecane, glycerol) were important to obtain high-quality vitrified islets. Modifications made to the experimental setup, such as installation of a cryostream and a specialized sample adapter, enabled the accelerated transfer of vitrified islets onto the sample stage, all within an uninterrupted cryogenic workflow. We demonstrated, for the first time, the feasibility of 2D XRF mapping of single islets of Langerhans, which provided element distributions of $\mathrm{K}, \mathrm{Ca}$, Fe and $\mathrm{Zn}$ with $500 \mathrm{~nm}$ spatial resolution. Since titanium was present as trace metal within the cryoloops, its XRF signal could be used for rapid defining of the scanning region. Additionally, we illustrated the feasibility of XRF cryotomography through entire islets, thereby providing additional information, e.g. their oblong shape in the cryoloop, the heterogeneous distribution of zinc and the presence of iron hot-spots. XRF tomographic reconstruction was hampered by presence of ice crystals causing diffraction. Further testing/optimisation of the cryofixation method would be required in this context. Pilot 2D cryo XRF mapping from islets of WT and KO mice under low (3mM) and high glucose (30 $\mathrm{mM})$ conditions did not reveal obvious changes in element distribution (and concentration) of $\mathrm{Ca}, \mathrm{K}$, 
Fe and $\mathrm{Zn}$. Mean element intensities (K, Ca, Mn, Fe, Ni, Cu, Zn, Sr, Br) of entire cluster islets did show variations between the different conditions which, however, may be due to natural variation. Examination of more islets would be required in this context. Nevertheless, the developed method proved to be valid and can be used for future studies on islets, as well as on other biological samples which can be captured in a cryoloop.

\section{Conflicts of interest}

There are no conflicts of interest to declare

\section{Acknowledgments}

We would like to thank Claire Naylor (Molecular dimensions, UK) for kindly providing the cryoloop test samples. MB was supported by a fellowship from the Clinical Research Fund, Cliniques Universitaires Saint-Luc, UCLouvain, Belgium. SJM is a post-doctoral researcher of the Flemish Research Foundation (FWO); he acknowledges funding under grant 12S5718N. JCJ is Research Director of the F.R.S.-FNRS, Belgium. We acknowledge DESY (Hamburg, Germany), a member of the Helmholtz Association HGF, for the provision of experimental facilities. Parts of this research were carried out at the PETRA III P06 beamline. This research was supported in part through the Maxwell computational resources operated at Deutsches Elektronen-Synchrotron (DESY). The research leading to this result has been supported by the project CALIPSOplus under the Grant Agreement 730872 from the EU Framework Programme for Research and Innovation HORIZON 2020. 


\section{Figure Captions}

Fig 1: a) Sample preparation workflow for SR XRF analysis of cryofrozen islets of Langerhans b) light microscopy image of steel pin with cryoloop inserted on top c) photograph of cryocap with steel pin and cryoloop (drawn).

Fig 2: Experimental setup at the P06 Hard X-ray Microprobe (PETRAIII, Hamburg) for analyzing islets of Langerhans under cryogenic conditions a) overview b) close-up and c) light microscope image of cryofrozen islet as observed by P06 beamline microscope.

Fig 3: Element distribution of K, Ca, Fe and Zn from islet 'C4' (KO, $3 \mathrm{mM}$ glucose). Element maps obtained from both silicon drift detectors (SDD) are provided (upper row: SDD no. 0 with $15^{\circ}$ offset; lower row: SDD no. 1 with orthogonal orientation). Intensity includes both $K_{\alpha}$ and $K_{\beta}$ fluorescent lines. Additionally, $K_{\alpha}$ fluorescent energy (in keV) is provided for each element. Cryoloop specifications: $300 \mu \mathrm{m}$ diameter, $20 \mu \mathrm{m}$ thickness. Scan parameters: $1 \mu \mathrm{m}$ pixel size, $100 \mathrm{~ms}$ dwell time/pixel. $\mathrm{K}_{\alpha}$ fluorescent line (in $\mathrm{keV}$ ) is provided for each element. Scale bar indicates $20 \mu \mathrm{m}$.

Fig 4: 2D XRF cryomapping of a single islet of Langerhans 'E4' (WT, 3mM glucose level). Cryoloop specifications: $200 \mu \mathrm{m}$ diameter; $20 \mu \mathrm{m}$ thickness. Intensity includes both $\mathrm{K}_{\alpha}$ and $\mathrm{K}_{\beta}$ fluorescent lines. Additionally, $\mathrm{K}_{\alpha}$ fluorescent energy (in $\mathrm{keV}$ ) is additionally provided for each element. Scan parameters: $500 \mathrm{~nm}$ pixel size, $150 \mathrm{~ms}$ dwell time/pixel (element map obtained from SDD no. 1, orthogonal geometry). Scale bar indicates $20 \mu \mathrm{m}$.

Fig 5: XRF cryotomography through same islet from Fig 3 'E4' (WT, 3 mM glucose). Sample was rotated every 3 degrees in discrete mode between 0 and $180^{\circ}$ (60 angles), dwell time was $50 \mathrm{~ms} / \mathrm{pixel}, 500 \mathrm{~nm}$ pixel size. Signal was obtained from SDD no. 1 (orthogonal orientation).

Fig 6: 2D XRF cryomapping of islets from WT/KO mice under low ( $3 \mathrm{mM})$ and high ( $30 \mathrm{mM})$ glucose levels. Intensity includes both $K_{\alpha}$ and $K_{\beta}$ fluorescent lines. Scan parameters: $1 \mu \mathrm{m}$ pixel size, $100 \mathrm{~ms}$ dwell time/pixel. Signal was obtained from SDD no. 1. Element maps of zinc and titanium are shown.

Fig 7: 2D XRF cryomapping of islets from WT/KO mice under low (3 mM) and high (30 mM) glucose levels. Intensity includes both $K_{\alpha}$ and $K_{\beta}$ fluorescent lines. Scan parameters: $1 \mu \mathrm{m}$ pixel size, $100 \mathrm{~ms}$ dwell time/pixel. Signal was obtained from SDD no. 1. Element maps of calcium and iron are shown.

Fig 8: Mean whole islet cluster intensities (in counts, cts) for the elements $\mathrm{P}, \mathrm{S}, \mathrm{Cl} \mathrm{K}, \mathrm{Ca}, \mathrm{Mn}, \mathrm{Fe}, \mathrm{Ni}, \mathrm{Cu}$,

$\mathrm{Zn}$, Se, Br and Rayleigh/Compton scatter for WT/KO islets with G3/G30 exposure (3/30 mmol glucose concentration). In graph a), intensities have been normalized both to number of pixels in the islet cluster (i.e. the area) and the Compton scatter peak (proportional to the electron density), whereas in graph b), only to the number of pixels (not taking into account differences in electron density between the different islets). 


\section{Bibliography}

1. R. E. Pratley and C. Weyer, Curr Diab Rep, 2002, 2, 242-248.

2. M. Bensellam, D. R. Laybutt and J. C. Jonas, Mol Cell Endocrinol, 2012, 364, 1-27.

3. M. Bensellam, J. C. Jonas and D. R. Laybutt, J Endocrinol, 2018, 236, R109-R143.

4. D. Pipeleers, Diabetologia, 1987, 30, 277-291.

5. F. C. Jonkers, J. C. Jonas, P. Gilon and J. C. Henquin, J Physiol, 1999, 520 Pt 3, 839-849.

6. G. Da Silva Xavier and G. A. Rutter, J Mol Biol, 2019, DOI: 10.1016/j.jmb.2019.08.005.

7. E. Lammert and P. Thorn, J Mol Biol, 2019, DOI: 10.1016/j.jmb.2019.10.032.

8. P. E. MacDonald, J. W. Joseph and P. Rorsman, Philos Trans R Soc Lond B Biol Sci, 2005, 360, 2211-2225.

9. J. C. Henquin, Diabetes Res Clin Pract, 2011, 93 Suppl 1, S27-31.

10. P. Gilon, H. Y. Chae, G. A. Rutter and M. A. Ravier, Cell Calcium, 2014, 56, 340-361.

11. S. O. Emdin, G. G. Dodson, J. M. Cutfield and S. M. Cutfield, Diabetologia, 1980, 19, 174-182.

12. C. P. Hill, Z. Dauter, E. J. Dodson, G. G. Dodson and M. F. Dunn, Biochemistry, 1991, 30, 917-924.

13. M. F. Dunn, Biometals, 2005, 18, 295-303.

14. J. B. Hansen, M. F. Tonnesen, A. N. Madsen, P. H. Hagedorn, J. Friberg, L. G. Grunnet, R. S. Heller, A. O. Nielsen, J. Storling, L. Baeyens, L. Anker-Kitai, K. Qvortrup, L. Bouwens, S. Efrat, M. Aalund, N. C. Andrews, N. Billestrup, A. E. Karlsen, B. Holst, F. Pociot and T. Mandrup-Poulsen, Cell Metab, 2012, 16, 449-461.

15. P. Chabosseau and G. A. Rutter, Arch Biochem Biophys, 2016, 611, 79-85.

16. L. Li, S. Bai and C. T. Sheline, Diabetes, 2017, 66, 551-559.

17. J. B. Hansen, L. R. B. Dos Santos, Y. Liu, K. J. Prentice, F. Teudt, M. Tonnesen, J. C. Jonas, M. B. Wheeler and T. Mandrup-Poulsen, J Mol Endocrinol, 2018, 61, 69-77.

18. R. M. Walter, Jr., J. Y. Uriu-Hare, K. L. Olin, M. H. Oster, B. D. Anawalt, J. W. Critchfield and C. L. Keen, Diabetes Care, 1991, 14, 1050-1056.

19. H. Zhang, C. Yan, Z. Yang, W. Zhang, Y. Niu, X. Li, L. Qin and Q. Su, J Trace Elem Med Biol, 2017, 40, 91-96.

20. G. Bjorklund, M. Dadar, L. Pivina, M. D. Dosa, Y. Semenova and J. Aaseth, Curr Med Chem, 2019, DOI: 10.2174/0929867326666190902122155.

21. M. Farooq, J Coll Physicians Surg Pak, 2019, 29, 253-257.

22. J. Yin, X. Wang, S. Li, Y. Zhu, S. Chen, P. Li, C. Luo, Y. Huang, X. Li, X. Hu, W. Yang, W. Bao, Z. Shan and L. Liu, Redox Biol, 2019, 24, 101172.

23. C. G. Schroer, I. Agapov, W. Brefeld, R. Brinkmann, Y. C. Chae, H. C. Chao, M. Eriksson, J. Keil, X. N. Gavalda, R. Rohlsberger, O. H. Seeck, M. Sprung, M. Tischer, R. Wanzenberg and E. Weckert, J. Synchrot. Radiat., 2018, 25, 1277-1290.

24. S. Chen, J. Deng, Y. Yuan, C. Flachenecker, R. Mak, B. Hornberger, Q. Jin, D. Shu, B. Lai, J. Maser, C. Roehrig, T. Paunesku, S. C. Gleber, D. J. Vine, L. Finney, J. VonOsinski, M. Bolbat, I. Spink, Z. Chen, J. Steele, D. Trapp, J. Irwin, M. Feser, E. Snyder, K. Brister, C. Jacobsen, G. Woloschak and S. Vogt, J. Synchrot. Radiat., 2014, 21, 66-75.

25. Y. Yang, F. Fus, A. Pacureanu, J. C. da Silva, W. De Nolf, C. Biot, S. Bohic and P. Cloetens, Anal. Chem., 2019, 91, 6549-6554.

26. B. De Samber, G. Silversmit, K. De Schamphelaere, R. Evens, T. Schoonjans, B. Vekemans, C. Janssen, B. Masschaele, L. Van Hoorebeke, I. Szaloki, F. Vanhaecke, K. Rickers, G. Falkenberg and L. Vincze, Journal of Analytical Atomic Spectrometry, 2010, 25, 544-553.

27. J. W. Pflugrath, Acta Crystallographica Section F-Structural Biology Communications, 2015, 71, 622-642. 
28. R. Schonherr, J. M. Rudolph and L. Redecke, Biological chemistry, 2018, 399, 751-772.

29. U. Boesenberg, C. G. Ryan, R. Kirkham, D. P. Siddons, M. Alfeld, J. Garrevoet, T. Nunez, T. Claussen, T. Kracht and G. Falkenberg, J. Synchrot. Radiat., 2016, 23, 1550-1560.

30. J. Garrevoet, B. Vekemans, P. Tack, B. De Samber, S. Schmitz, F. E. Brenker, G. Falkenberg and L. Vincze, Anal. Chem., 2014, 86, 11826-11832.

31. C. G. Schroer, C. Baumbach, R. Dohrmann, S. Klare, R. Hoppe, M. Kahnt, J. Patommel, J. Reinhardt, S. Ritter, D. Samberg, M. Scholz, A. Schropp, F. Seiboth, M. Seyrich, F. Wittwer and G. Falkenberg, in Proceedings of the 12th International Conference on Synchrotron Radiation Instrumentation, eds. Q. Shen and C. Nelson, Amer Inst Physics, Melville, 2016, vol. 1741.

32. F. Seiboth, A. Schropp, M. Scholz, F. Wittwer, C. Rodel, M. Wunsche, T. Ullsperger, S. Nolte, J. Rahomaki, K. Parfeniukas, S. Giakoumidis, U. Vogt, U. Wagner, C. Rau, U. Boesenberg, J. Garrevoet, G. Falkenberg, E. C. Galtier, H. J. Lee, B. Nagler and C. G. Schroer, Nat. Commun., 2017, 8, 5 .

33. M. Bensellam, Y. C. Shi, J. Y. Chan, D. R. Laybutt, H. Chae, M. Abou-Samra, E. G. Pappas, H. E. Thomas, P. Gilon and J. C. Jonas, Diabetologia, 2019, 62, 2273-2286.

34. C. G. Schroer, O. Kurapova, J. Patommel, P. Boye, J. Feldkamp, B. Lengeler, M. Burghammer, C. Riekel, L. Vincze, A. van der Hart and M. Kuchler, Appl. Phys. Lett., 2005, 87, 3.

35. C. G. Schroer, P. Boye, J. M. Feldkamp, J. Patommel, D. Samberg, A. Schropp, A. Schwab, S. Stephan, G. Falkenberg, G. Wellenreuther and N. Reimers, Nucl. Instrum. Methods Phys. Res. Sect. A-Accel. Spectrom. Dect. Assoc. Equip., 2010, 616, 93-97.

36. V. A. Sole, E. Papillon, M. Cotte, P. Walter and J. Susini, Spectrochimica Acta Part B-Atomic Spectroscopy, 2007, 62, 63-68.

37. P. P. Bruyant, J Nucl Med, 2002, 43, 1343-1358.

38. B. De Samber, S. Vanblaere, R. Evens, K. De Schamphelaere, G. Wellenreuther, F. Ridoutt, G. Silversmit, T. Schoonjans, B. Vekemans, B. Masschaele, L. Van Hoorebeke, K. Rickers, G. Falkenberg, I. Szaloki, C. Janssen and L. Vincze, Powder Diffraction, 2010, 25, 169-174.

39. M. C. Foster, R. D. Leapman, M. X. Li and I. Atwater, Biophys J, 1993, 64, 525-532. 

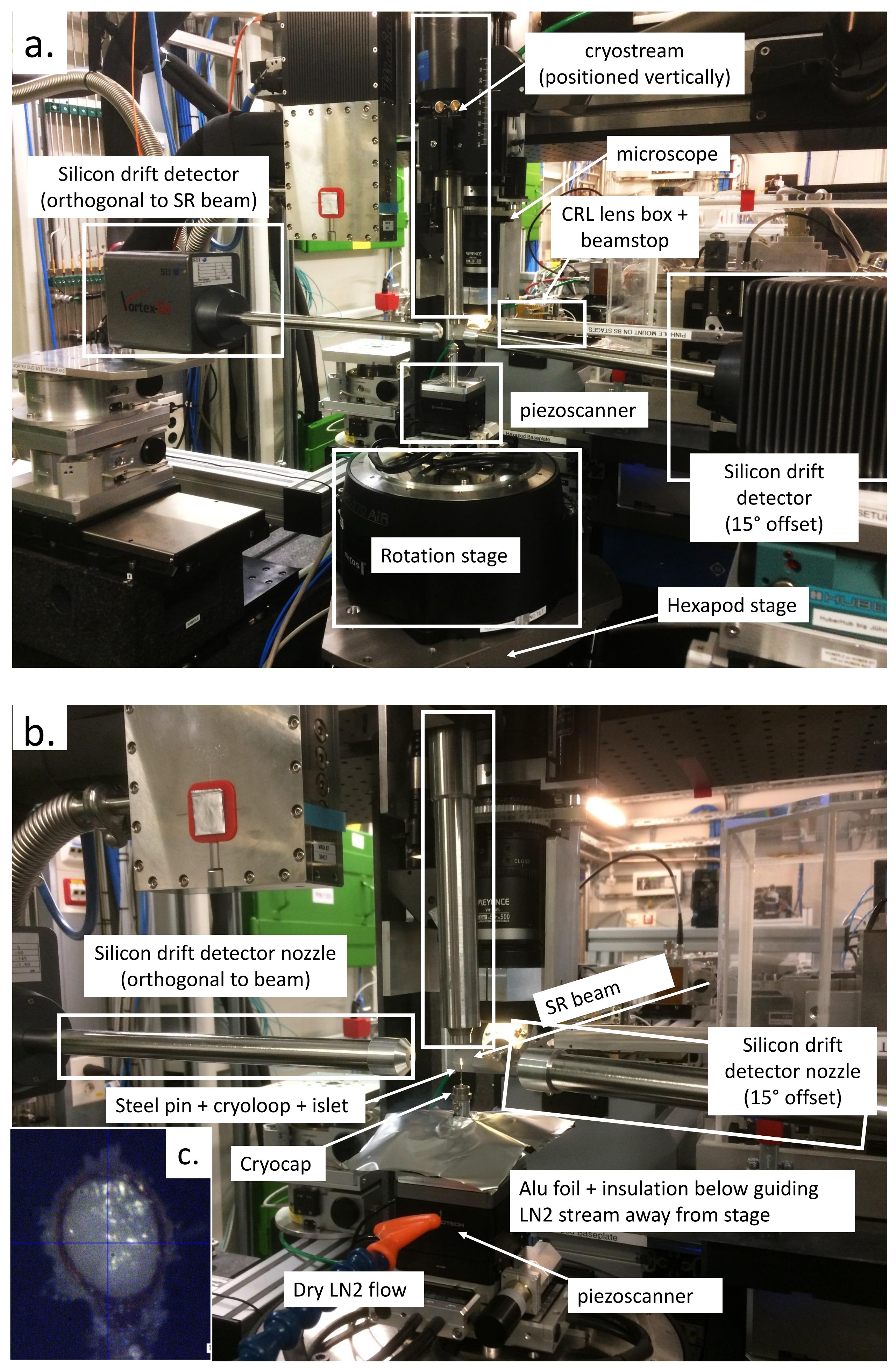


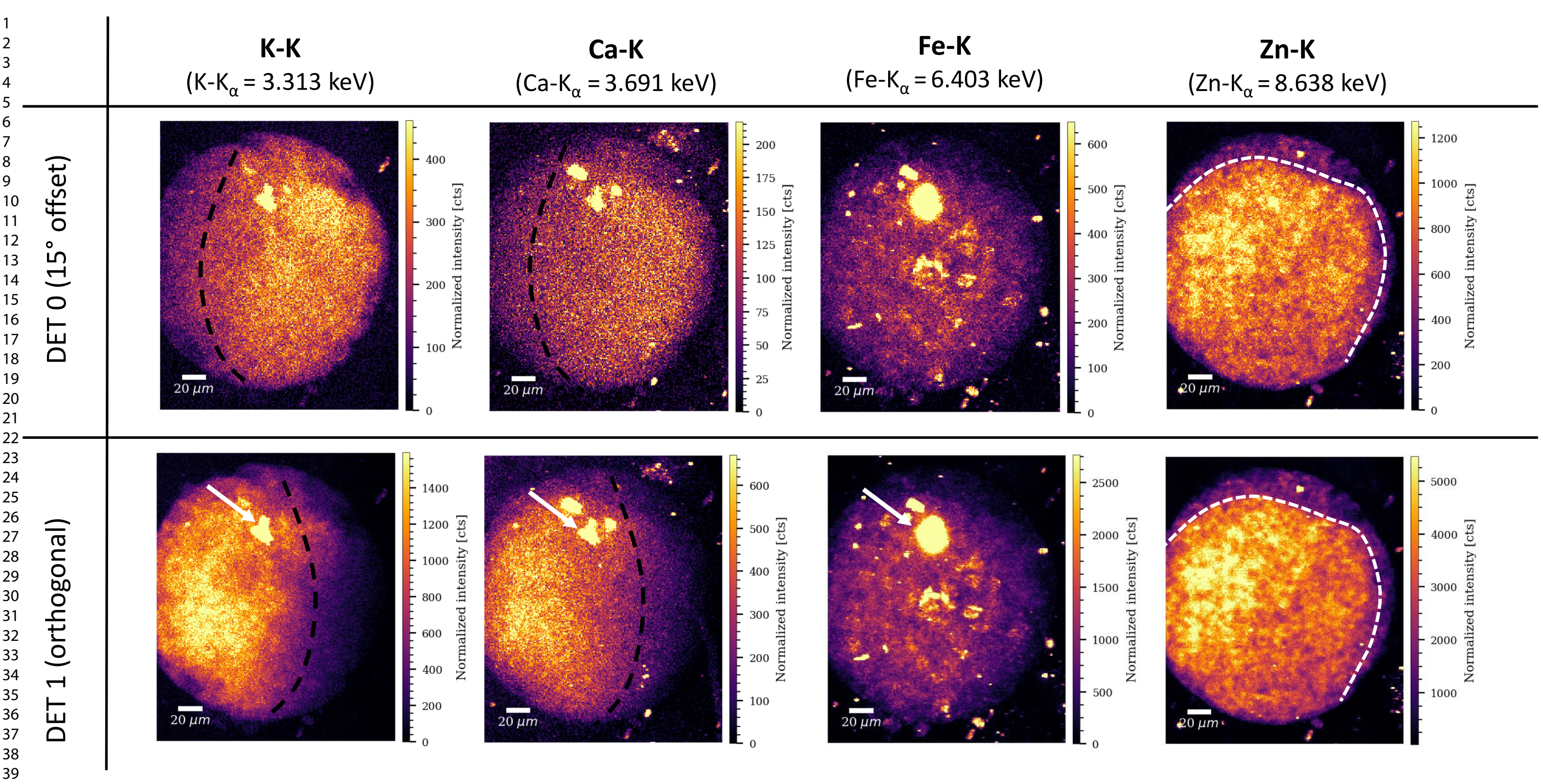



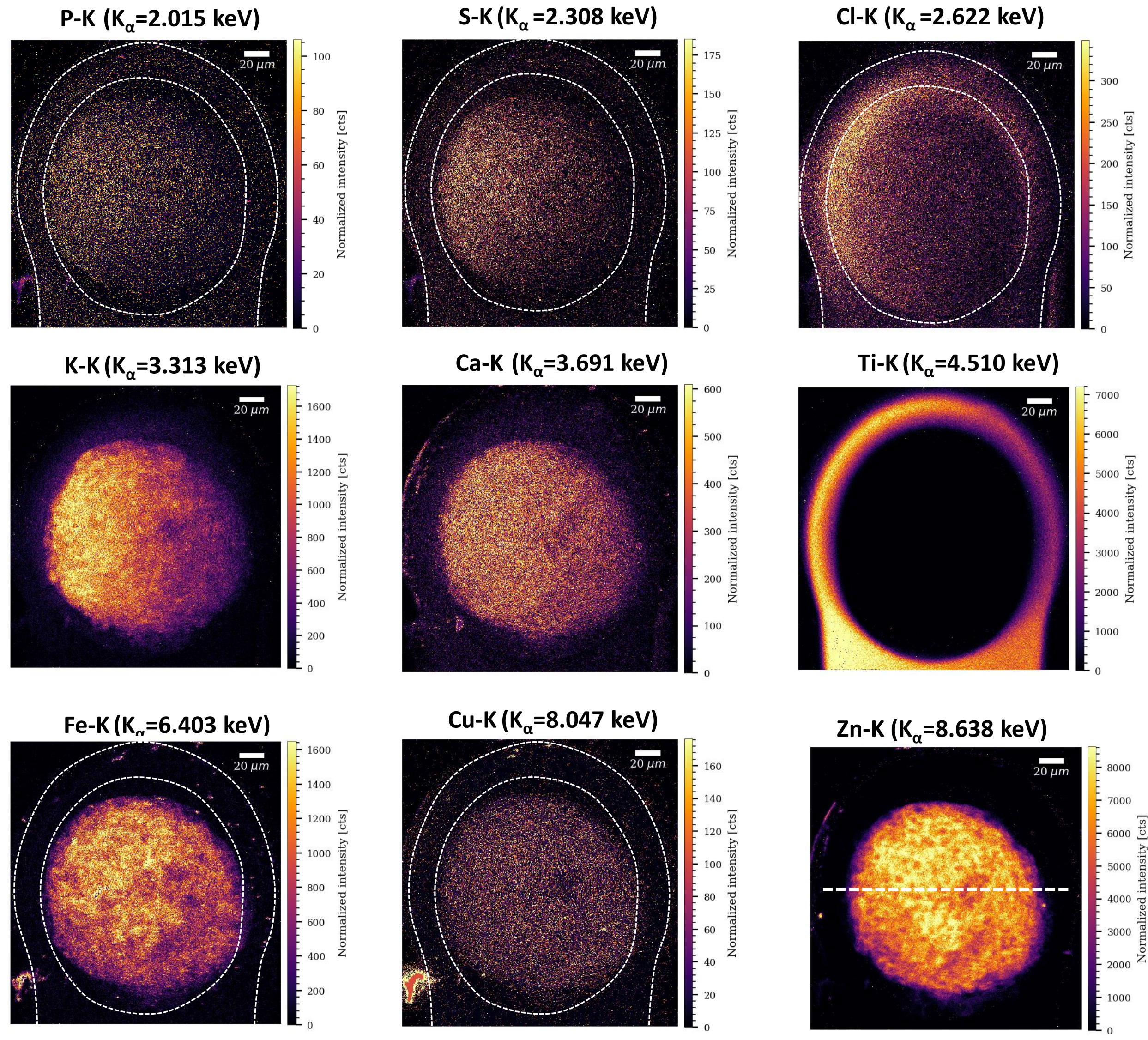


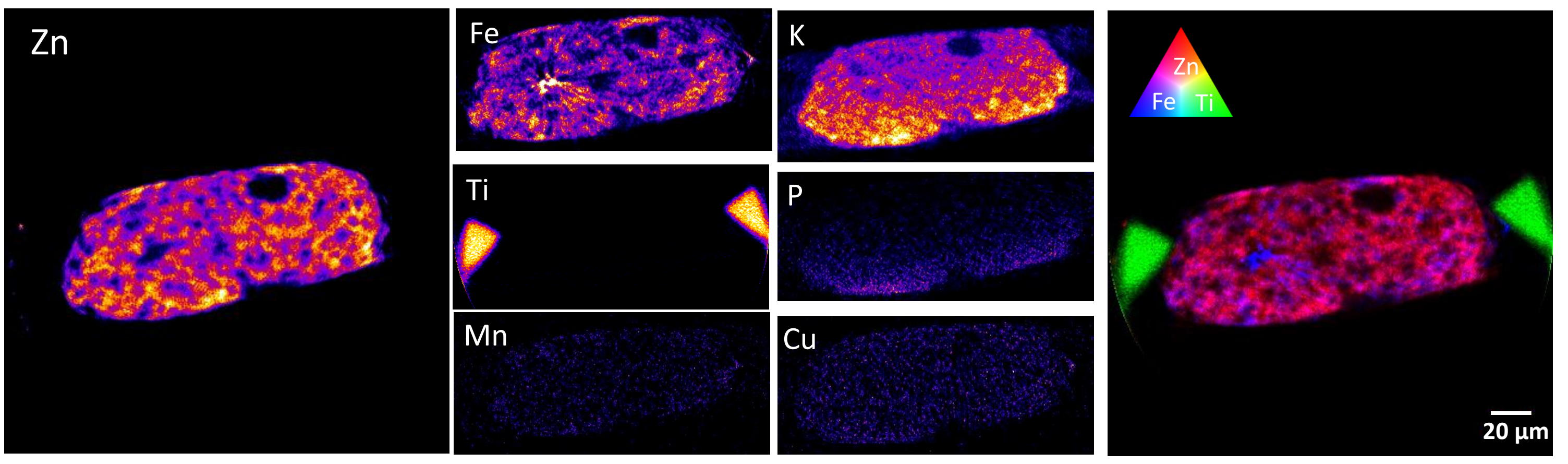




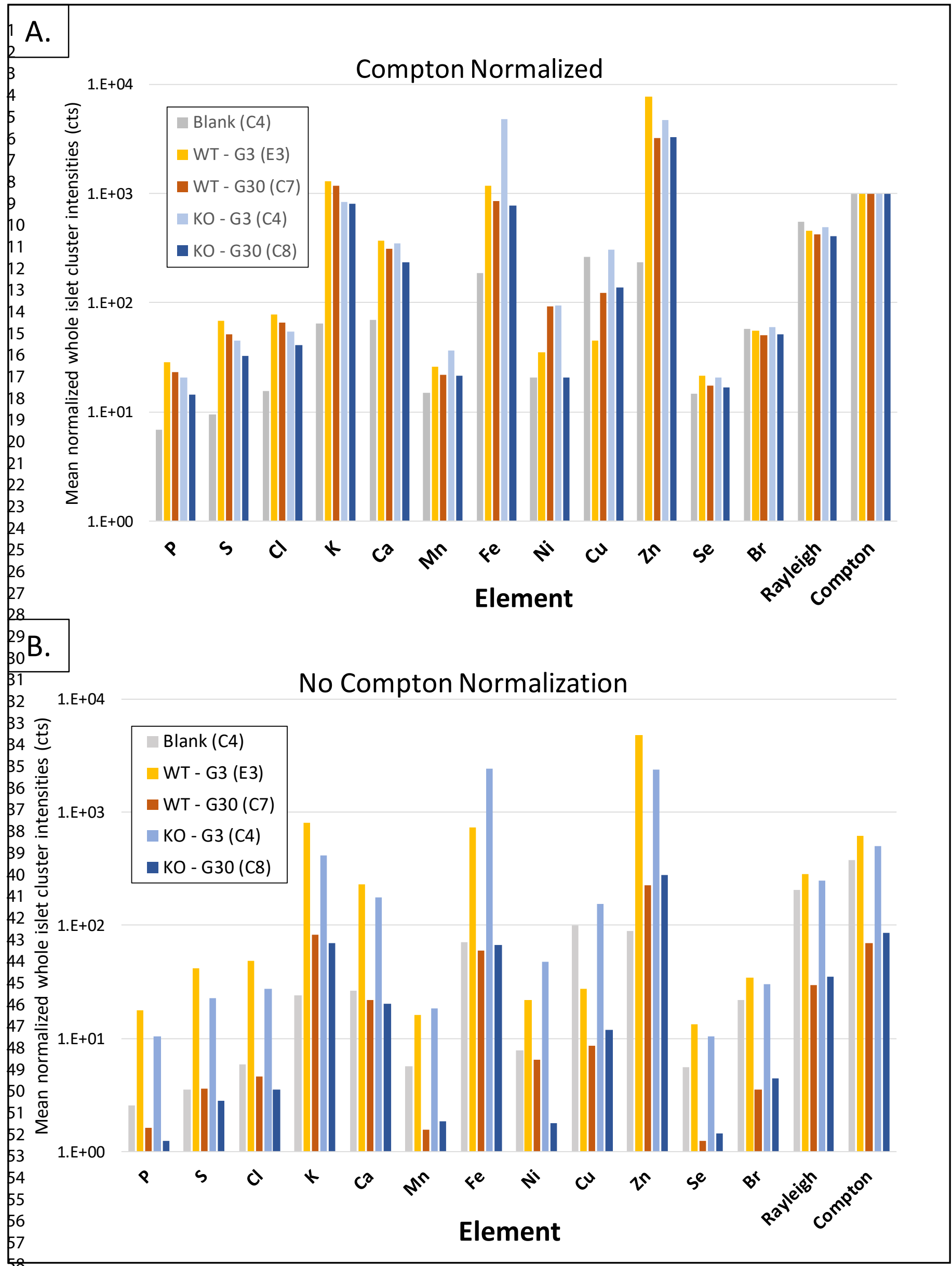

